\title{
Word predictability affects saccade length in Chinese reading: An evaluation of the dynamic-adjustment model
}

\author{
Yanping Liu $^{1} \cdot$ Siyuan Guo ${ }^{1}$ Lei Yu ${ }^{1} \cdot$ Erik D. Reichle $^{2}$
}

Published online: 31 July 2017

(C) Psychonomic Society, Inc. 2017

\begin{abstract}
How does a word's within-sentence predictability influence saccade length during reading? An eye-movement experiment manipulating the predictability of target words indicates that, relative to low-predictability target words, highpredictability targets elicit longer saccades to themselves. Simulations using computational models that respectively instantiate the targeting of saccades to default locations (Yan, Kliegl, Richter, Nuthmann, \& Shu in Journal of Experimental Psychology, 63, 705-725, 2010) versus the dynamic adjustment of saccade length (Liu, Reichle, \& Li in Journal of Experimental Psychology Learning Memory and Cognition, 41, 1229-1236, 2015, Journal of Experimental Psychology: Human Perception and Performance, 42, 1008-1025, 2016) indicate that the latter model provides a more accurate and parsimonious account of saccade-targeting behavior in Chinese reading. The implications of these conclusions are discussed with respect to current models of eye-movement control during reading and the necessity to explain eye movements in languages as different as Chinese versus English.
\end{abstract}

Keywords Chinese reading · Dynamic-adjustment model · Eye-movement control · Word-predictability effect

Yanping Liu

liuyp33@mail.sysu.edu.cn

Erik D. Reichle

e.reichle@soton.ac.uk

1 Department of Psychology, Guangdong Provincial Key Laboratory of Social Cognitive Neuroscience and Mental Health, Sun Yat-sen University, No. 132, Waihuan East Road, Guangzhou Higher Education Mega Center, Guangzhou 510006, People's Republic of China

2 Department of Psychology, University of Southampton, Southampton SO17 1BJ, UK

\section{Introduction}

Does a word's within-sentence predictability determine where the word will be fixated during reading? For example, in reading "The cat chased the...", English speakers will likely anticipate the next word to be "mouse," but does this expectation affect where a reader will look at the word if it is actually fixated? According to prevailing accounts of saccade targeting, in writing systems where words are demarcated by clear boundaries, predictability only influences a reader's decision about whether or not to fixate a word (i.e., predictable words are skipped more often than unpredictable words; Balota, Pollatsek, \& Rayner, 1985; Ehrlich \& Rayner, 1981; Rayner, Slattery, Drieghe, \& Liversedge, 2011; Rayner \& Well, 1996) but should not affect where the word is fixated when it is not skipped (cf., Lavigne, Vitu, \& d'Ydewalle, 2000; Rayner, Binder, Ashby, \& Pollatsek, 2001; see Staub, 2015, for a review). By this account, words that are not skipped tended to be fixated to the left of center, on the preferred-viewing locations (PVL; Rayner, 1979). Although predicable words are also skipped more often in Chinese (e.g., Rayner, Li, Juhasz, \& Yan, 2005), relatively little is known how predictability influences saccade targeting in Chinese. This is unfortunate because there are reasons to suspect that saccade targeting may be different in unspaced languages like Chinese, and that variables like predictability may influence where words are fixated.

For example, early work indicated that the normal-shaped initial-fixation distributions or PVL curves observed in English tended to be uniform in Chinese, suggesting that Chinese readers do not move their eyes to default targets (e.g., see Tsai \& McConkie, 2003, Fig. 8.1; Yang \& McConkie, 1999, Table 10.4). This view was challenged by a corpus analysis (Yan, Kliegl, Richter, Nuthmann, \& Shu, 2010; see also Yan \& Kliegl, 2016), which suggested why PVL curves are flat: Although words that are fixated once tend to be the fixated near 
their PVLs, words that are fixated twice tend to be initially fixated near their beginning and then fixated closer to their end. Based on this finding, Yan et al. proposed a flexible or defaulttargeting hypothesis ${ }^{1}$ : Words that are segmented in the parafovea are the recipients of single fixation near their PVL, whereas words that are not segmented tend to be fixated near their beginning to allow an additional fixation to aid its identification. This account, however, provides a circular explanation of saccade targeting because a word that is by chance the recipient of a single fixation near its PVL might be identified more rapidly, thereby eliminating the needs for a second fixation $(\mathrm{Li}, \mathrm{Liu}, \&$ Rayner, 2011; see Liu, Reichle, \& Li, 2015, 2016).

An alternative account of saccade targeting in Chinese is that saccade lengths are adjusted dynamically as a function of lexical processing demands (Liu et al., 2015, 2016; Liu, Huang, Gao, \& Reichle, 2017; Liu, Huang, Li, \& Gao, 2017). By this dynamic-adjustment account, Chinese readers lengthen an impending saccade if the preprocessing of the parafoveal word is easy. Although simulations using implemented versions of the dynamic-adjustment and defaulttargeting accounts indicate that the former accounted for saccade-targeting behavior better than the latter (see Liu et al., 2016), both accounts are limited in that they currently only posit roles for lexical processing in saccade targeting, with lexical variables affecting the relative ease of processing $\operatorname{word}_{N+1}$ from word affecting where word $_{N+1}$ will be fixated. These variables include the frequencies of $\operatorname{word}_{N}$ and $\operatorname{word}_{N+}$ ${ }_{1}$, the launch-site fixation location on $\operatorname{word}_{N}$, and whether or not normal preview of $\operatorname{word}_{N+1}$ is available from $\operatorname{word}_{N}$. One important variable that has not been examined is predictability; although there is ample evidence that predictability can facilitate the preprocessing of a word being viewed parafoveally through the extraction of its visual, orthographic, phonological, and semantic features (Balota et al., 1985; Schotter, Lee, Reiderman, \& Rayner, 2015; White, Rayner, \& Liversedge, 2005; see Schotter, Angele, \& Rayner, 2012, for a review), it is not known whether predictability also modulates saccade length. This article therefore examines how word predictability influences saccade length by first providing evidence that it does and then comparing how well the default-targeting versus dynamic-adjustment models account for this evidence.

\footnotetext{
$\overline{1}$ The name given to this "flexible" hypothesis is unfortunate because, although it does allow some degree of flexibility in saccade-target selection, this choice is limited to a small number of default targets - the beginning or middle of the next word, or when the next word is skipped, the beginning of the following word. We therefore use "default-targeting" to describe this class of models and reserve "dynamic" for those models that posit the adjustment of saccade length rather than saccade-target selection, per se.
}

\section{Empirical method}

\section{Participants}

Thirty native Chinese-speaking students from Sun Yat-sen University were paid 20 yuan to participate. All participants had a normal or corrected-to-normal vision, were naive about the purpose of the experiment, and gave prior informed consent.

\section{Apparatus}

Eye movements were recorded by an SR Research Ltd. EyeLink 1000-plus eye tracker, sampling at 1,000 Hz. Participants' heads were stabilized in a tower setup that minimized head movements with forehead and chin rests, being seated approximately $58 \mathrm{~cm}$ from a 27 -in. LED monitor (ASUS, PG27AQ, 2,560 × 1,440 pixel resolution, 144-Hz refresh rate). Sentences were displayed in the vertical center of the screen using 30-point white Song font characters (1 character $\approx 1^{\circ}$ of visual angle) on a black background.

\section{Materials and experimental design}

Target words consisted of 80 pairs of high-predictability $(M=$ $0.85 ; S D=0.12)$ and low-predictability $(M=0 ; S D=0)$ twocharacter words having similar meanings (Contemporary Chinese Dictionary, 2012). Predictability norms were collected from a separate sample of 15 native Chinese speakers asked to predict target word identities using their preceding sentence contexts; high-predictability words were predicted more than low-predictability words $(t=65.52, p<0.001)$. One target word of either type was then embedded near the center of one of the sentence frames (Fig. 1). Another 18 native Chinese speakers evaluated the naturalness of the sentences, with no difference between high- and low-predictability condition $(t=-1.08, p>0.28)$. The frequency and complexity (i.e., stroke number) of target words and their characters were controlled (Table 1), with no differences between high- and low-predictability condition ( $p s>0.33$ ). During the experiment, each participant read each sentence frame once and read equal numbers of sentences in each condition in a counterbalanced design.

\section{Procedure}

At the beginning of the experiment, the eye tracker was calibrated and validated using a 3-point calibration routine. Each trial began with a drift-check in the middle of the screen followed by a $1^{\circ} \times 1^{\circ}$ fixation box displayed at the location of the first character of the sentence (to check calibration), with a sentence appearing after the fixation box was successfully fixated. If the fixation box was not fixated or the drift check indicated more 


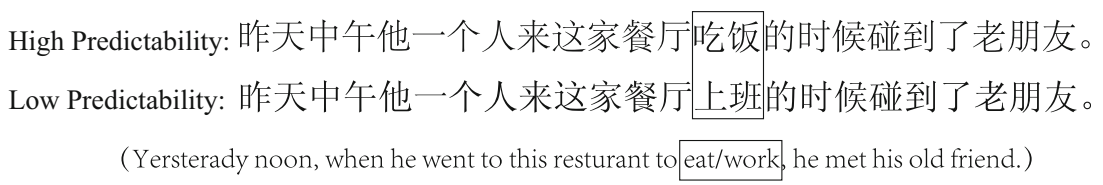

Fig. 1 Examples of the Chinese sentences used in the experiment and their English translations. (For illustrative purposes, the target words are indicated by solid boxes)

than $0.4^{\circ}$ error, then the participant was recalibrated. Viewing was binocular, but only right eye movements were recorded. Each participant first read 20 practice sentences (excluded from our analysis) and then read the 80 experimental sentences in random order. Participants were instructed to read silently with comprehension and to press a response button (Microsoft SideWinder Game Pad) to answer comprehension questions (after one-third of sentences) and initiate trials.

\section{Empirical results}

\section{Data preparation}

Trials containing an eye blink on or immediately preceding or following target words were excluded from analyses, resulting in $1.3 \%$ of the total trials being removed.

\section{Comprehension accuracy}

Participants correctly answered $95 \%$ of the comprehension questions and comprehension was not affected by targetword predictability ( $p=0.869)$.

\section{Eye-movement measures}

To examine how target-word predictability affected saccade targeting, our primary analyses focused on two saccade-length measures: (1) progressive-saccade length (PSL), or the lengths of the initial saccades from the pre-target region, irrespective of whether they actually resulted in a fixation on the target word; (2) incoming-saccade length (ISL), or the subset of progressive saccades that actually resulted in a fixation on the target word. To control for any possible effects of saccade launch site (e.g., limited perceptual span), our analyses were restricted to saccades launched from the two-character pretarget region. And to facilitate comparison of our results with the literature, we also examined how target-word predictability affected: (3) skipping probability (SP), or the probability of skipping the target word; (4) first-fixation duration (FFD), or the duration of the initial target-word fixation during first-pass reading; (5) gaze duration (GD), or the sum of first-pass target-word fixations; and (6) total-viewing time (TT), or the sum of all target-word fixations.

For each measure, linear mixed-effect models (or generalized linear mixed models for SP) were fitted using the measure as the dependent variable and target-word predictability as the design factor (coded as sum contrasts: 0.5 vs. -0.5 for high and low predictability), so that each intercept estimates the grand mean of a given dependent variable and the regression coefficients estimates the differences between factor levels. (Launchsite fixation duration was also included as a covariate when analyzing progressive and incoming saccade lengths.) To maximize the generalizability of our analyses and avoid over-parameterization, the models used the parsimonious randomeffects structure, with appropriate variance and covariance components due to participants, items, and their slopes (Bates, Kliegl, Vasishth, \& Baayen, 2015). Models were fitted using the lme4 package (ver. 1.1-13; Bates, Maechler, Bolker, $\&$ Walker, 2015) and $p$-values were estimated by using the lmerTest package (ver. 2.0-33) in R (ver. 3.4.1).

As can be seen by inspecting the means (Table 2) and the (generalized) linear mixed-effect models (Table 3), both progressive and incoming saccade lengths were longer for highpredictability than low-predictability target words (PSL: $b=$ $0.21, S E=0.08, t=2.73, p=0.008 ;$ ISL: $b=0.09, S E=0.05, t$

Table 1 Properties of target words

\begin{tabular}{|c|c|c|c|c|}
\hline \multirow[t]{2}{*}{ Variables } & \multicolumn{2}{|c|}{ Word predictability } & \multicolumn{2}{|c|}{ Inferential statistics } \\
\hline & High & Low & $T$ & $P$ \\
\hline Word predictability & 0.85 & 0.00 & 65.52 & $<0.001$ \\
\hline Word frequency (per million) & 63.03 & 60.86 & 0.03 & 0.97 \\
\hline First character frequency (per million) & $1,400.38$ & $1,261.09$ & 0.49 & 0.62 \\
\hline Second character frequency (per million) & $1,222.20$ & $1,523.28$ & -0.98 & 0.33 \\
\hline First character stroke number & 7.96 & 7.89 & 0.14 & 0.89 \\
\hline Second character stroke number & 8.00 & 8.33 & -0.66 & 0.51 \\
\hline
\end{tabular}


Table 2 Progressive-saccade length (PSL), incoming-saccade length (ISL), skipping probability (SP), first-fixation duration (FFD), gaze duration (GD), and total-viewing time (TT), as a function of target-word predictability

\begin{tabular}{|c|c|c|c|c|c|c|}
\hline Word predictability & PSL & ISL & SP & FFD & GD & TT \\
\hline High & $3.79(0.18)$ & $2.57(0.07)$ & $0.44(0.03)$ & $254(8)$ & $263(9)$ & $307(12)$ \\
\hline Low & $3.58(0.17)$ & $2.52(0.06)$ & $0.39(0.03)$ & $275(8)$ & $292(9)$ & 364 (17) \\
\hline
\end{tabular}

Standard errors are shown in parentheses

Note: Saccade length is measured in character spaces and fixation-duration measures are in milliseconds

$=1.88, p=0.061)$. (The arbitrary exclusion of saccades that under- or overshot the target words in the more restrictive analysis of incoming saccades attenuated the effect of predictability.) Replicating Liu, Huang, Gao, and Reichle (2017), longer launch-site fixation durations elicited shorter progressive $(b=-0.001, S E=0.0004, t=-1.73, p=0.084)$ and incoming saccades $(b=-0.001, S E=0.0003, t=-2.26, p=$ $0.025)$. Finally, the remaining analyses also replicated previous findings (e.g., see Rayner et al., 2005): High-predictability words were skipped more often $(b=0.24, S E=0.10, t=2.63$, $p=0.012$ ) and the recipients of fewer, shorter fixations (FFD: $b=-23.27, S E=6.18, t=-3.77, p<0.001$ GD: $b=-31.65$, $S E=7.49, t=-4.23, p<0.001 ;$ TT: $b=-61.28, S E=12.06, t$ $=-5.08, p<0.001)$ than low-predictability words.

\section{Simulation method}

To better understand our results, we examined how target-word predictability affects saccade length using the default-targeting and dynamic-adjustment models in the simulations reported below (for detailed model descriptions, see Liu et al., 2016). The basic method used with both models is as follows: During each Monte-Carlo trial, a launch-site was first sampled from a uniformly distributed two-character pre-target region; a saccade target (Simulation 1) or saccade length (Simulation 2) was then selected and some amount of saccadic error was introduced. This process was repeated 10,000 times for each model.

\section{Simulation 1: Default-targeting model}

According to this model, if a target word is segmented, a saccade is directed towards its center; otherwise, a saccade is directed towards its beginning, allowing the word to be refixated. The model does not specify precisely how this happens, but instead uses Yan et al.'s (2010) assumptions about the relationship between word segmentation and saccade targeting to estimate the probability of target word segmentation from how often it was fixated, using four mutually exclusive and exhaustive saccade behaviors: (1) refixating pretarget region; (2) fixating target word and then moving the eyes from this word (presumably because it was segmented in the parafoveal); (3) fixating and then refixating target word (presumably because it was not segmented in the parafovea); and (4) skipping target word. The polynomial regression functions (Eq. 1) were fit to each possible saccade launch site, with the constraint that the probabilities of the four types of eyemovement behaviors from each saccade launch site summed to 1 . To account for any effect of predictability, these functions were fit separately for each predictability condition. In Eq. 1, $x$ represents the distance (in character spaces) between the pretarget launch site and the leftmost edge of target word, and $\mathrm{k}_{2}$, $\mathrm{k}_{1}$, and $\mathrm{k}_{0}$ respectively represent the coefficients of the $2^{\circ}, 1^{\circ}$, and $0^{\circ}$ polynomials.

$p(x)=k_{2} x^{2}+k_{1} x+k_{0}$

The estimated probabilities were then used to specify saccade targets as follows: (1) a saccade to refixate the pre-target region caused the eyes to move towards its center; (2) the successful parafoveal segmentation of the target word caused the eyes to move towards its center; (3) the failure to parafoveally segment the target word caused the eyes to move towards its beginning (i.e., the center of its first character); (4) a saccade to skip the target word caused the eyes to move towards the beginning of post-target word (i.e., the center of its first character). Finally, variance was added to the saccade

Table 3 LMM inferential statistics for progressive-saccade length (PSL), incoming-saccade length (ISL), skipping probability (SP), first-fixation duration (FFD), gaze duration (GD), and total-viewing time (TT), as a function of target-word predictability

\begin{tabular}{llllll}
\hline Predictors & PSL & ISL & SP & FFD & GD \\
\hline Intercept & $3.82^{* * * *}$ & $2.69^{* * *}$ & $-0.36^{* *}$ & $264.69^{* * * *}$ & $277.37^{* * * *}$ \\
Target predictability (high) & $0.21^{* *}$ & $0.09^{\dagger}$ & $0.24^{*}$ & $-23.27^{* * *}$ & $-31.65^{* * * *}$ \\
Launch-site fixation duration & $-0.001^{\dagger}$ & $-0.001^{*}$ & - & - & $-61.28^{* * *}$ \\
\hline
\end{tabular}

Note: Significance levels: $\dagger p<0.1 ; * p<0.05 ; * * p<0.01$; and $* * * p<0.001$ 


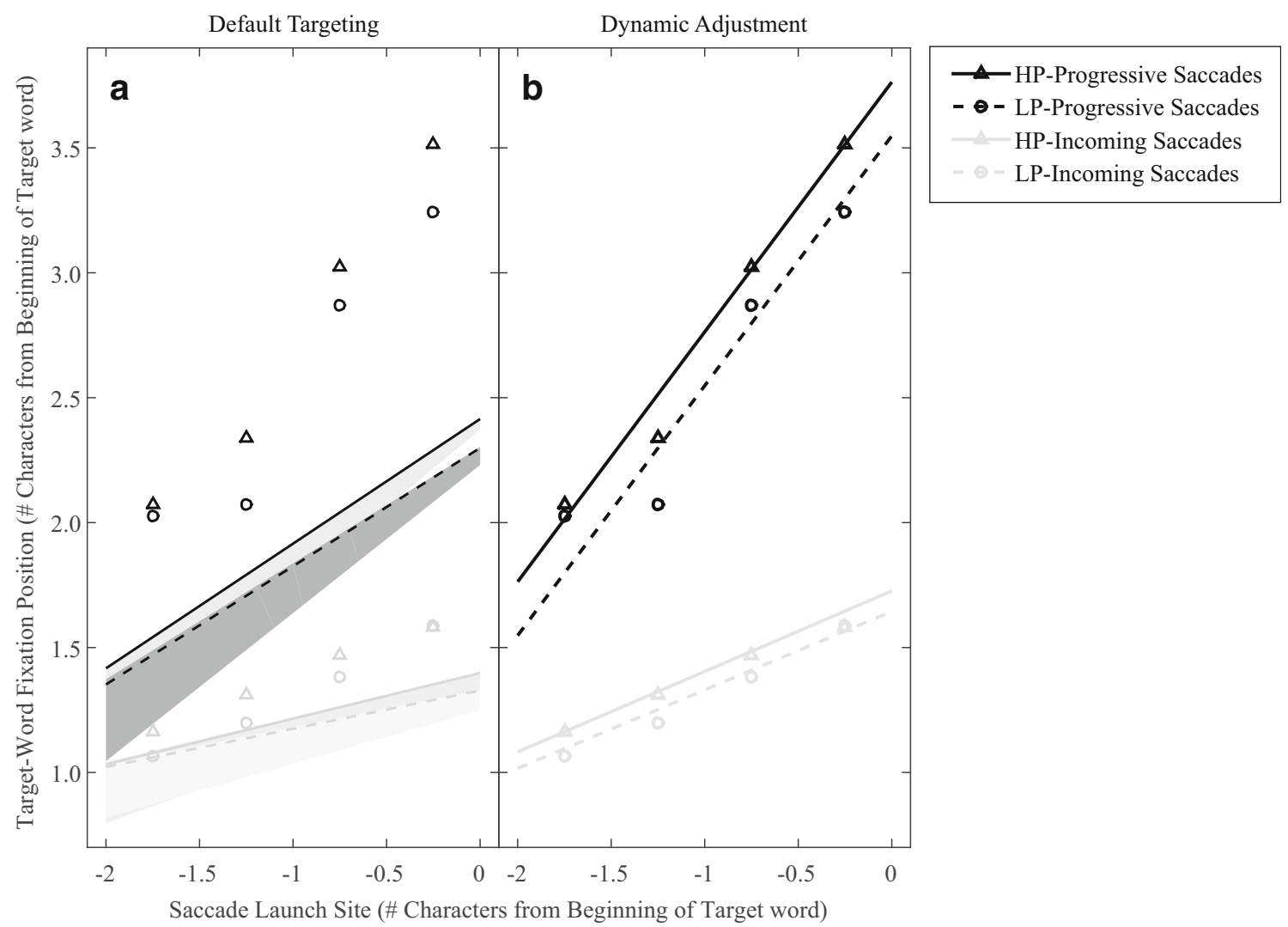

Fig. 2 The predicted relationship between the saccade launch site and the subsequent fixation landing site (in character spaces) generated by the: (a) default-targeting model (Simulation 1), and (b) dynamic-adjustment model (Simulation 2). The symbols show the observed means averaged within each launch-distance bin. The black and gray lines represent the simulated progressive and incoming saccades, respectively, and both

target to simulate saccadic error, which was sampled from a Gaussian distribution with $\mu=0$, and the values of $\sigma$ being selected to fit the empirical fixation-position distributions of incoming saccades on target word (see Appendix). The results of Simulation 1 are discussed below, in comparison to Simulation 2.

\section{Simulation 2: Dynamic-adjustment model}

According to this model, Chinese readers adjust their saccade lengths as a function of the amount of parafoveal processing completed. To implement this assumption, it was also necessary to use a simplifying assumption - that saccade length is a linear function of parafoveal preprocessing. To do this, the amount of target-word preprocessing completed from the pre-target region was sampled from a gamma distribution having a shape parameter, $\alpha$, and a scale parameter, $\beta$, as described by Eq. 2 .

preprocessing $=\operatorname{gamma}(\alpha, \beta)$

Using this equation, the amount of target-word preprocessing (as determined by the value of $\alpha$ ) was modulated by the launch sites and landing sites are aligned to the beginning of target word. The shaded regions in panel (a) demarcates the default-targeting model's performance across its full domain (i.e., between the two most extreme cases in which the target word is never vs. always segmented in the parafovea). $H P$ high-predictability, $L P$ low-predictability

predictability of target word, as specified by Eq. 3. (The modest effect of saccade launch-site distance and duration on preprocessing is ignored for the sake of simplicity.) In this equation, $\eta_{0}$ represents the minimal value of $\alpha$ and $\eta_{1}$ modulates the influence of target-word predictability on $\alpha$.

$\alpha=\eta_{0}+\eta_{1}$ predictability

The final assumption is that saccade length (in character spaces) is linearly related to preprocessing, as specified by Eq. 4 , where $\lambda$ scales this relationship. In contrast to Simulation 1, saccadic error is intrinsic to Simulation 2, with saccade length variability determined by $\beta$.

$$
\begin{aligned}
& \text { length }=\lambda \text { preprocessing } \\
& =\lambda \operatorname{gamma}(\alpha, \beta) \\
& =\operatorname{gamma}\left(\eta_{0}+\eta_{1} \text { predictability, } \lambda \beta\right)
\end{aligned}
$$

\section{Simulation results}

To facilitate comparison, the simulation and empirical results are displayed in Figs. 2 and 3. Figure 2 shows how well the 


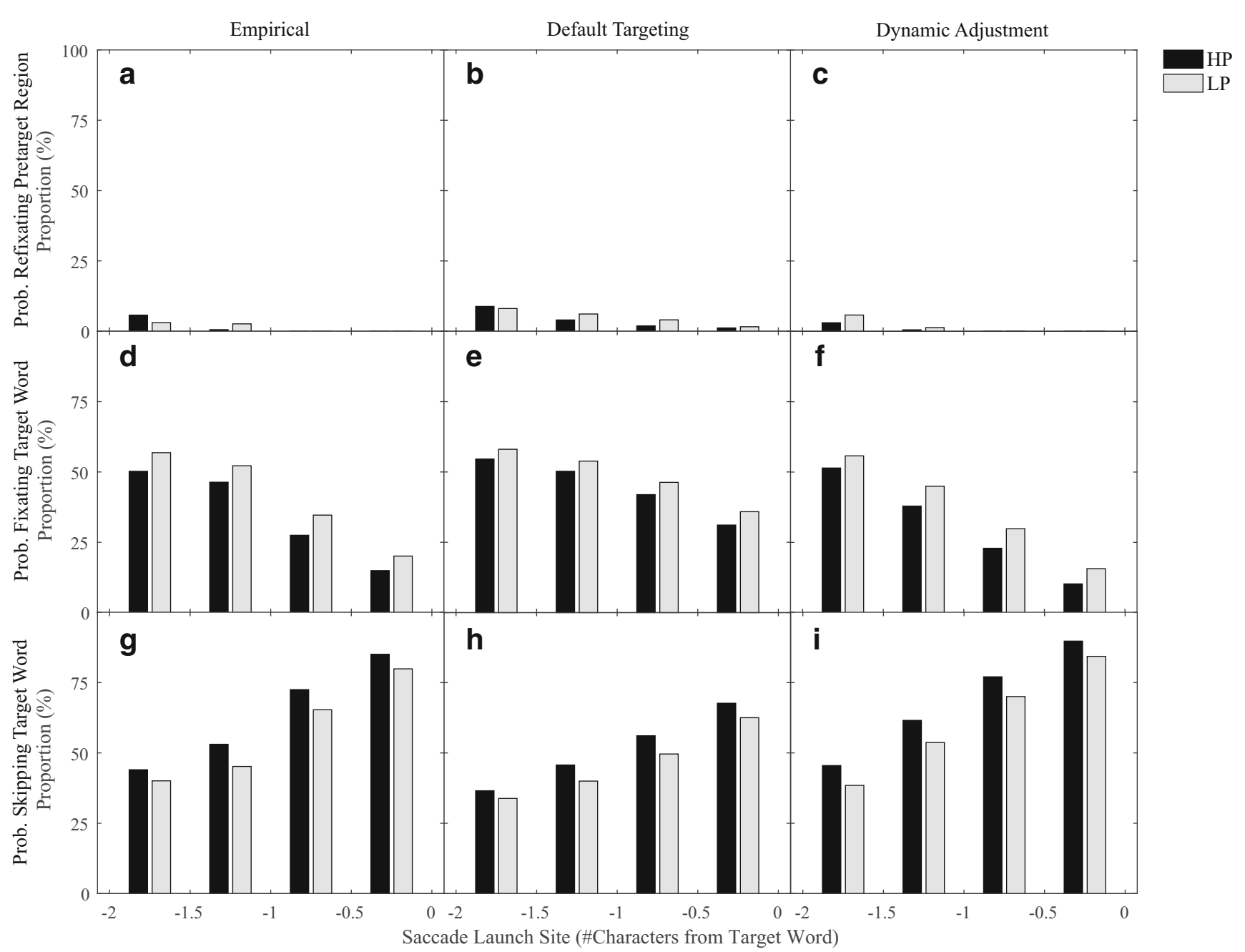

Fig. 3 Mean observed and simulated probabilities of refixating the pre-target region (panels a-c), fixating the target word (panels d-f), and skipping the target word (panels g-i). $H P$ high-predictability, $L P$ low-predictability

models fit the quantitative relationships observed between pre-target saccade launch sites and fixation landing sites on high- and low-predictability target words. The dynamicadjustment model provides a better quantitative fit than the default-targeting model for both progressive (DefaultTargeting: $M S E=7.04 \times 10^{-1}$; Dynamic-Adjustment: $M S E$ $=1.85 \times 10^{-2}$ ) and incoming saccades (Default-Targeting: $M S E=3.31 \times 10^{-2}$; Dynamic-Adjustment: $M S E=5.06 \times$ $\left.10^{-4}\right)$. The poor fit of the default-targeting model cannot be explained by suboptimal estimates of word-segmentation probabilities (see the shaded regions of Fig. 2A, which show the model's fits across the full domain of estimates).

Figure 3 shows the mean observed and simulated probabilities of refixating the pre-target region, and of fixating and skipping the target word. The dynamic-adjustment model again provides a better quantitative fit of the data than the default-targeting model: (1) refixating pre-target region (Default-Targeting: $M S E=1.00 \times 10^{-3}$; DynamicAdjustment: $M S E=2.05 \times 10^{-4}$ ); (2) fixating target word
(Default-Targeting: $M S E=1.12 \times 10^{-2}$; DynamicAdjustment: $M S E=2.60 \times 10^{-3}$ ); and (3) skipping target word (Default-Targeting: $M S E=1.62 \times 10^{-2}$; DynamicAdjustment: $M S E=2.90 \times 10^{-3}$ ).

Table 4 The best-fitting parameters for the default-targeting model (Simulation 1)

\begin{tabular}{lllll}
\hline Saccade type & $k_{2}$ & $k_{1}$ & $k_{0}$ & $\sigma$ \\
\hline $\begin{array}{l}\text { High-predictability } \\
\quad \text { refixating pretarget region }\end{array}$ & 0.052 & 0.068 & 0.016 & 0.638 \\
$\quad$ fixating center of target word & -0.083 & -0.405 & 0.042 & \\
$\quad$ fixating beginning of target word & -0.004 & -0.018 & -0.004 & \\
Low-predictability & & & & \\
$\quad$ refixating pretarget region & 0.005 & -0.015 & -0.006 & 0.698 \\
$\quad$ fixating center of target word & -0.078 & -389 & 0.095 & \\
$\quad$ fixating beginning of target word & -0.021 & -0.065 & -0.009 & \\
\hline
\end{tabular}




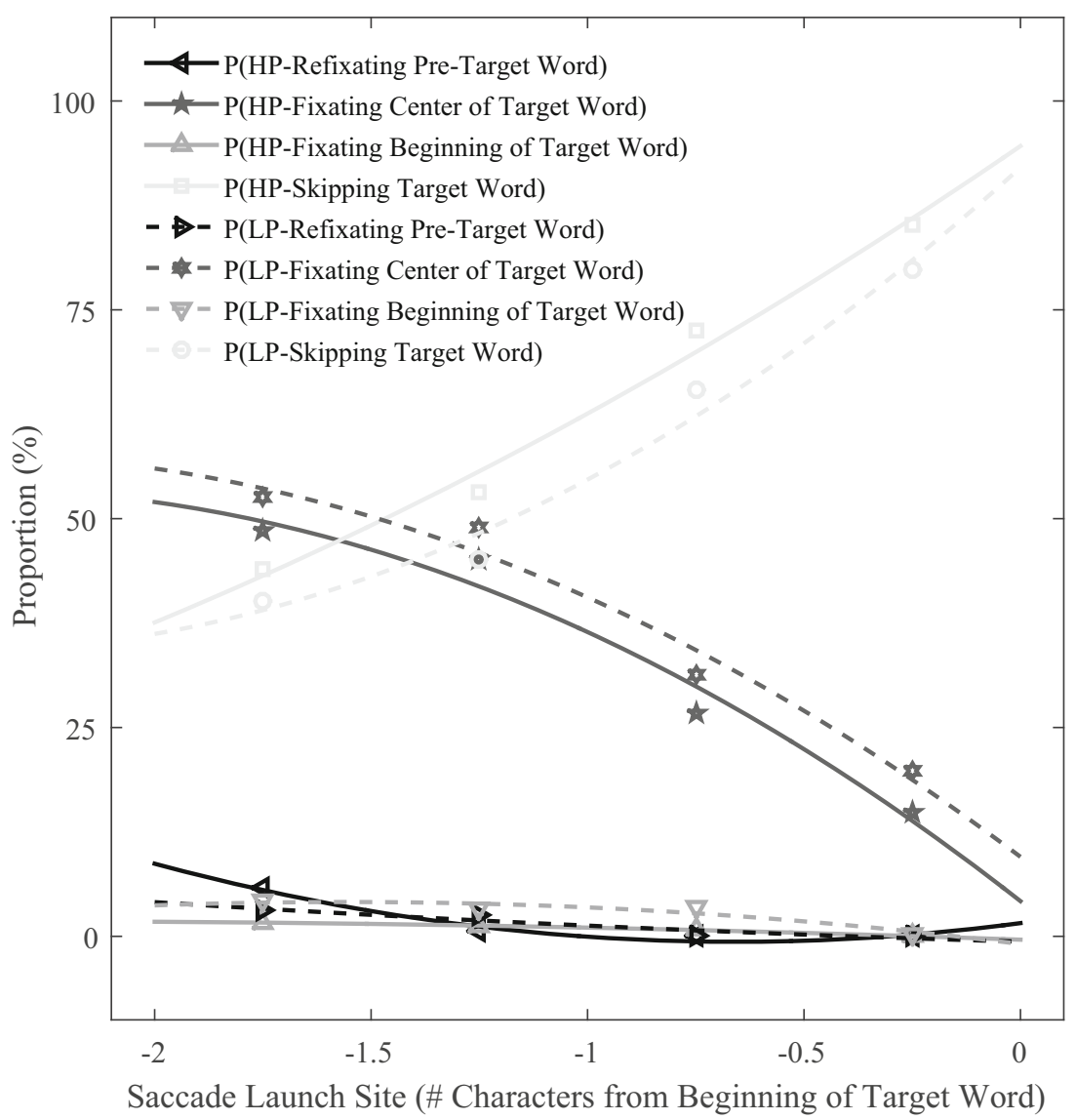

Fig. 4 The observed (symbols) and estimated (lines) probabilities of refixating pre-target word, fixating the center of target word (i.e., single fixation), fixating the beginning of target word (i.e., first-of-multiple fixations), and skipping target word as a function of the predictability of target word. $H P$ high-predictability, $L P$ low-predictability

\section{General discussion}

This article reported the results of an eye-movement experiment and two simulations examining how the predictability of a word in the parafovea influences where a reader's eyes will be directed. The empirical results replicate prior findings that predictable words tend to be skipped more often and the recipients of fewer, shorter fixations (Rayner et al., 2005), but additionally demonstrates that readers move their eyes further into predictable than unpredictable words. This latter is important because the evidence for word-predictability effects on fixation positions in alphabetic languages has been mixed: Although a few experiments have reported fixation landingsite distributions are further to the right in predictable than unpredictable words (Lavigne et al., 2000; see also Kennedy, Murray, \& Boissiere, 2004; McDonald \& Shillcock, 2003a, 2003b), other experiments have failed to replicate this result (Rayner et al., 2001; Vainio, Hyönä, \& Pajunen, 2009). Furthermore, the "standard" explanation of this result, when it is observed, is that readers are more likely to skip predictable words, resulting in an increased proportion of the saccades falling short of their intended target, thereby shifting the fixation landing-site distribution further into predictable words (e.g., Rayner et al., 2001).

However, the simulations reported in this article suggest an alternative account - one in which parafoveal lexical processing difficulty informs decisions about how far to move the eyes during reading (Liu et al., 2015). Current and prior simulations using a explicit computational version of this dynamic-adjustment account indicate that its assumptions are sufficient to reproduce the patterns of saccade-targeting eye-movement behaviors that are observed in Chinese reading, and that this account is more accurate and parsimonious than the one provided by an implemented version of the default-targeting account (Liu et al., 2016; Liu, Huang, Gao et al., 2017). The current efforts add to these prior demonstrations, however, by providing evidence that a supra-lexical variable- a word's withinsentence predictability - modulates the immediate decisions about where the word will be fixated. As such, the 
result provides another challenge to models of eyemovement control in reading that posit default targeting (e.g., E-Z Reader: Reichle, Pollatsek, \& Rayner, 2012; SWIFT: Engbert, Nuthmann, Richter, \& Kliegl, 2005). More specifically, this challenge entails specifying precisely how the dynamic adjustment of saccade length accords with the possible use of default saccade targets in the context of reading languages as different as Chinese and English.

Author note All authors contributed equally to this work. This research was supported by the grants from the National Natural Science Foundation of China (31500890), by the Fundamental Research Funds for the Central Universities (17wkpy64), and by U.S. National Institutes of Health Grant HD075800.

\section{Appendix}

Simulation 1 parameters As Eq. 1 shows, polynomial regression functions were used to estimate the probabilities of observing the four different types of saccades using the method of least squares. Because these probabilities summed to 1 for each saccade launch site, only the probabilities associated with three saccades types were estimated. Finally, the values of $\sigma$, which control saccadic-error variability, were chosen to maximize the goodness-of-fit to the empirical fixation-position distributions of incoming saccades in the high- and low-predictability conditions separately (MSE $=0.033$ and 0.031 , respectively). Table 4 lists the best-fitting parameters. Figure 4 shows that these parameters accurately describe the empirical data (i.e., probability of refixating pre-target region: $M S E=2.47 \times 10^{-5}$; probability of fixating target-word center: $M S E=5.55 \times 10^{-4}$; probability of fixating target-word beginning: $M S E=1.25 \times$ $10^{-5}$ ). Simulation 1 thus required 20 free parameters.

Simulation 2 parameters The expected value of Eq. 4 is $\lambda \beta\left(\eta_{0}\right.$ $+\eta_{1}$ predictability), or the predicted value using the mean first progressive saccade length from the pre-target word. Two parameter groups, $\lambda \beta \eta_{1}$ and $\lambda \beta \eta_{0}$, are coefficients for a regression equation for progressive saccade length, using target-word predictability (i.e., low-predictability $=-0.5$, high-predictability $=$ 0.5 ) as the predictor variable. Because the variance associated with Eq. 4 (i.e., associated with saccadic error) is given by the quantity $\lambda^{2} \beta^{2}\left(\eta_{1}\right.$ predictability $\left.+\eta_{0}\right)$, the parameter pair $\lambda \beta$ can be estimated using the empirical fixation distributions on highand low-predictability target words. The final parameters used to simulate the high-predictability condition were: $\eta_{0}=9.08 ; \eta_{1}=$ -0.40 ; and $\lambda \beta=-0.54$; the parameters for the low-predictability condition were: $\eta_{0}=5.89 ; \eta_{1}=-0.26$; and $\lambda \beta=-0.35$. Simulation 2 thus required six free parameters.

\section{References}

Balota, D. A., Pollatsek, A., \& Rayner, K. (1985). The interaction of contextual constraints and parafoveal visual information in reading. Cognitive Psychology, 17, 364-390.

Bates, D., Kliegl, R., Vasishth, S., \& Baayen, H. (2015). Parsimonious mixed models. arXiv:1506.04967.

Bates, D., Mächler, M., Bolker, B., \& Walker, S. (2015). Fitting linear mixed-effects models using lme4. Journal of Statistical Software, 67, $1-48$

Ehrlich, S. F., \& Rayner, K. (1981). Contextual effects on word perception and eye movements during reading. Journal of Verbal Learning and Verbal Behavior, 20, 641-655.

Engbert, R., Nuthmann, A., Richter, E. M., \& Kliegl, R. (2005). SWIFT: A dynamical model of saccade generation during reading. Psychological Review, 112, 777-813.

Kennedy, A., Murray, W. S., \& Boissiere, C. (2004). Parafoveal pragmatics revisited. European Journal of Cognitive Psychology, 16, 128 153.

Lavigne, F., Vitu, F., \& d'Ydewalle, G. (2000). The influence of semantic context on initial eye landing sites in words. Acta Psychologica, 104, 191-214.

Li, X., Liu, P., \& Rayner, K. (2011). Eye movement guidance in Chinese reading: Is there a preferred viewing location? Vision Research, 51, $1146-1156$.

Liu, Y., Huang, R., Gao, D., \& Reichle, E. D. (2017). Further tests of a dynamic-adjustment account of saccade targeting during the reading of Chinese. Cognitive Science. doi:10.1111/cogs.12487

Liu, Y., Huang, R., Li, Y., \& Gao, D. (2017). The word frequency effect on saccade targeting during Chinese reading: Evidence from a survival analysis of saccade length. Frontiers in Psychology, 8, 116.

Liu, Y., Reichle, E. D., \& Li, X. (2015). Parafoveal processing affects outgoing saccade length during the reading of Chinese. Journal of Experimental Psychology Learning Memory and Cognition, 41, 1229-1236.

Liu, Y., Reichle, E. D., \& Li, X. (2016). The effect of word frequency and parafoveal preview on saccade length during the reading of Chinese. Journal of Experimental Psychology: Human Perception and Performance, 42, 1008-1025.

McDonald, S. A., \& Shillcock, R. C. (2003a). Eye movements reveal the on-line computation of lexical probabilities during reading. Psychological Science, 14, 648-652.

McDonald, S. A., \& Shillcock, R. C. (2003b). Low-level predictive inference in reading: The influence of transitional probabilities on eye movements. Vision Research, 43, 1735-1751.

Rayner, K. (1979). Eye guidance in reading: Fixation location within words. Perception, 8, 21-30.

Rayner, K., Binder, K. S., Ashby, J., \& Pollatsek, A. (2001). Eye movement control in reading: Word predictability has little influence on initial landing positions in words. Vision Research, 41, 943-954.

Rayner, K., Li, X., Juhasz, B. J., \& Yan, G. (2005). The effect of word predictability on the eye movements of Chinese readers. Psychonomic Bulletin \& Review, 12, 1089-1093.

Rayner, K., Slattery, T. J., Drieghe, D., \& Liversedge, S. P. (2011). Eye movements and word skipping during reading: Effects of word length and predictability. Journal of Experimental Psychology: Human Perception and Performance, 37, 514-528.

Rayner, K., \& Well, A. D. (1996). Effects of contextual constraint on eye movements in reading: A further examination. Psychonomic Bulletin \& Review, 3, 504-509.

Reichle, E. D., Pollatsek, A., \& Rayner, K. (2012). Using E-Z Reader to simulate eye movements in non-reading tasks: A unified framework for understanding the eye-mind link. Psychological Review, 119, 155-185. 
Schotter, E. R., Angele, B., \& Rayner, K. (2012). Parafoveal processing in reading. Attention, Perception \& Psychophysics, 74, 5-35.

Schotter, E. R., Lee, M., Reiderman, M., \& Rayner, K. (2015). The effect of contextual constraint on parafoveal processing in reading. Journal of Memory and Language, 83, 118-139.

Staub, A. (2015). The effect of lexical predictability on eye movements in reading: Critical review and theoretical interpretation. Language and Linguistics Compass, 9, 311-327.

Tsai, J. L., \& McConkie, G. W. (2003). Where do Chinese readers send their eyes? In J. Hyönä, R. Radach, \& H. Deubel (Eds.), The mind's eye: Cognitive and applied aspects of eye movement research (pp. 159-176). Oxford: Elsevier.

Vainio, S., Hyönä, J., \& Pajunen, A. (2009). Lexical predictability exerts robust effects on fixation duration, but not on initial landing position during reading. Experimental Psychology, 56, 66-74.
White, S. J., Rayner, K., \& Liversedge, S. P. (2005). The influence of parafoveal word length and contextual constraint on fixation durations and word skipping in reading. Psychonomic Bulletin \& Review, 12, 466-471.

Yan, M., \& Kliegl, R. (2016). CarPrice versus CarpRice: word boundary ambiguity influences saccade target selection during the reading of Chinese sentences. Journal of Experimental Psychology: Learning, Memory, and Cognition, 42, 1832-1838.

Yan, M., Kliegl, R., Richter, E. M., Nuthmann, A., \& Shu, H. (2010). Flexible saccade-target selection in Chinese reading. Quarterly Journal of Experimental Psychology, 63, 705-725.

Yang, H.-M., \& McConkie, G. W. (1999). Reading Chinese: Some basic eye movement characteristics. In H. C. Chen (Ed.), Reading Chinese script: A cognitive analysis (pp. 207-222). Mahwah: Erlbaum. 